\title{
Study for Simultaneous Degradation of 2nd and 3rd Harmonics Radiation using a Random Rectenna Array
}

\author{
By Yoshiyuki FuJINO, Yuusuke YoshIOKA and Yasushi KoBAYASHI \\ Toyo University, Kawagoe, Japan
}

(Received June 21st, 2015)

\begin{abstract}
Rectenna array is a power reception device of Satellite Solar Power System (SSPS). In the ground sites of SSPS, there are huge number of rectenna elements (rectenna array) that is a device which was equipped with rectifying circuits and receiving antennas. A diode is used in a rectenna, which is nonlinear element, higher harmonic components are generated. Once these higher harmonic waves are reradiate to free space, the electromagnetic interference problems may occur to existing communication system. One method to decrease the higher harmonics is usage of a random rectenna array, which is a method to change the harmonics phase from rectenna elements randomly. This composes a rectenna array which does not have strong directivity on specific direction. In this paper, we proposed a method to simultaneous degradation of 2nd and 3rd harmonics at the same time by adjusting phase of each rectenna elements. In the calculation, degradation level of random rectenna array becomes more than $-15 \mathrm{~dB}$ in 100 elements compared to the conventional case, both in omni-directional element and CMSA element.
\end{abstract}

Key Words: Space Solar Power System, Rectenna, Harmonics, Random Rectenna Array

\section{Nomenclature}

$\begin{array}{lll}f_{0} & : & \text { fundamental frequency } \\ n f_{0} & : & \text { n-th harmonics frequency } \\ F_{m}(\theta) & : & \text { array factor of } \mathrm{m} \text {-th harmonics frequency } \\ g_{m}(\theta) & : & \text { element pattern of } \mathrm{m} \text {-th harmonics. } \\ E_{m}(\theta) & : & \text { Electric field of } \mathrm{m} \text {-th harmonics } \\ A & : & \text { evaluate function } \\ w_{m} & : \text { weight of } \mathrm{m} \text {-th harmonics generation }\end{array}$

\section{Introduction}

Space Solar Power System (SSPS) is a system to generate the energy by huge solar panel in the space, and its energy is transmitted by the wireless power transmission technology to the ground sites. In the ground sites, there are huge number of rectenna elements (rectenna array) that is a device which was equipped with rectifying circuits and receiving antennas.

Rectenna uses diodes to rectify reception power, which is nonlinear device. So, it generates not only DC (direct current) and fundamental wave $\left(f_{0}\right)$, but also higher harmonics wave $\left(n f_{0}\right)$. Normally, these harmonics are removed by the filter mounted in the rectenna, but, remaining component through the filter is reradiated to the free space, which is shown in Fig. 1. Its unwanted wave may cause the radio interference problem ${ }^{1)}$ to the other radio systems, because the harmonics frequencies are allocated to other radio systems. So, it is important problem to the quantitative evaluation of unwanted radiation.

Measurement method and quantitative evaluation method of a higher harmonics form a rectenna element is established by reference 2,3). Also, Radchneko ${ }^{4}$ shows fundamental investigation of degradation of reradiation from rectenna harmonics varying phase of rectenna array ${ }^{4}$, but it is not confirmed experientially.

The authors were proposed random rectenna array that feeding phase is randomly changed and observed degradation of $2 \mathrm{nd}$ harmonics level to be $-17 \mathrm{~dB}$ compared to conventional (in-phase) rectenna array in 100 elements linear array,6). However, harmonics generated in the ractenna array is not only 2nd harmonics, but also, 3rd or more order harmonics, is generated. So, simultaneous harmonics degradation method of 2nd and 3rd order is considered in this paper.

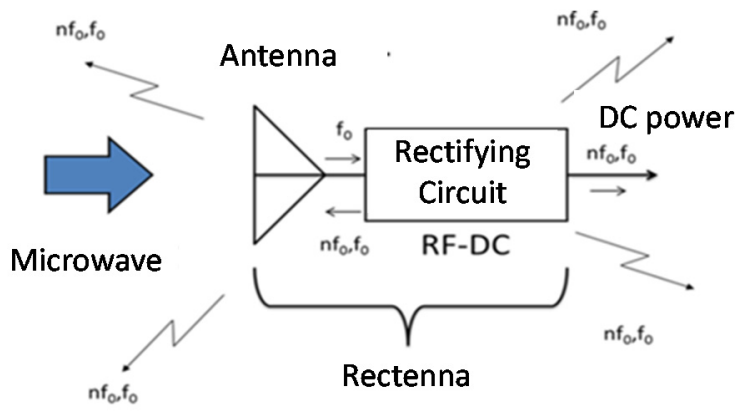

Fig. 1. Configuration of rectenna and harmonics re-radiation.

\section{Random Rectenna Array and Its Directivity}

Figure 2 left shows conventional rectenna array. This array has large grating lobe in harmonic frequencies, because each rectenna elements generates harmonics with in-phase.

To solve this problem, we proposed random rectenna array that changed electrical length between antenna and rectifying 
circuit randomly indicated in Fig. 2 right. It has not grating lobe in specific direction and has averaged directivity in the harmonics frequencies.

At first, one dimensional random rectenna array is calculated. Figure 3 shows a coordinate system. Array factor $F_{m}(\theta)$ of rectenna array in $\mathrm{m}$-th order harmonics is shown in following equation:

$$
F_{m}(\theta)=\sum_{n=1}^{n} a_{m, n} e^{j \varphi_{m, n}} e^{\left[j(n-1) k_{m} d \sin \theta\right]}
$$

where $a_{m, n}$ is a amplitude of $n$ element in $m$-th harmonics, $\varphi_{m, n}$ is random phase of $n$ element in $m$-th harmonics, $k_{m}$ is wave number at $m$-th harmonics frequencies. Also, $d$ is an interelement distance and set it to $\mathrm{d}=0.7 \lambda$, due to highest rectenna efficiency $^{7}$.

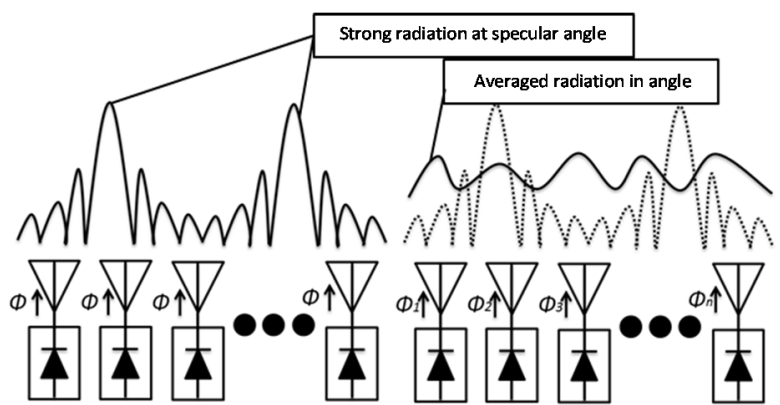

Fig. 2. Conventional rectenna array (left) and Random rectenna array (right).

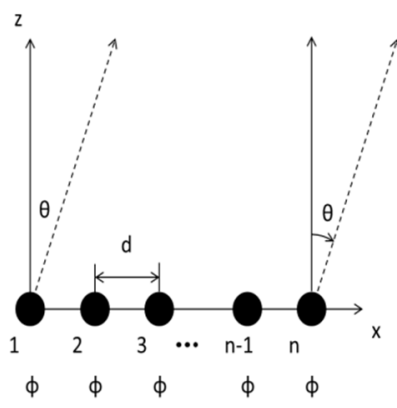

(a) Conventional rectenna array

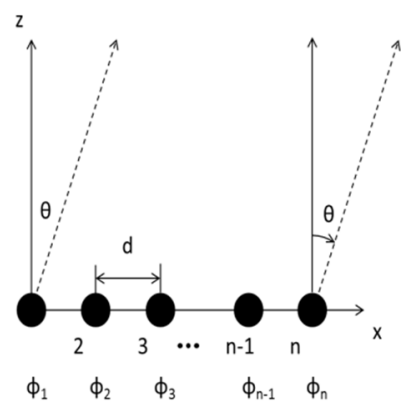

(b) Random rectenna array
Fig. 3. n-elements linear rectenna array.

To calculate harmonics degradation level of random rectenna array, we suppose omni-directivity of antenna element for the convenience. Electric field directivity $E_{m}(\theta)$ of random rectenna array is shown in next equation:

$$
E_{m}(\theta)=g_{m}(\theta) \times F_{m}(\theta)
$$

where $g_{m}(\theta)$ is element antenna pattern.

To achieve random phase, one way is change the feed line length randomly in each element. In this case each elements has different length, so it is easy way to get random phase. But, RFDC conversion efficiency of rectenna element will be change in the feed line length. So, it is not suitable for random rectenna array.

Then we will prepare some length of feedlines with fixed phase difference without affecting efficiency. By combining these elements random rectenna array will be realized.

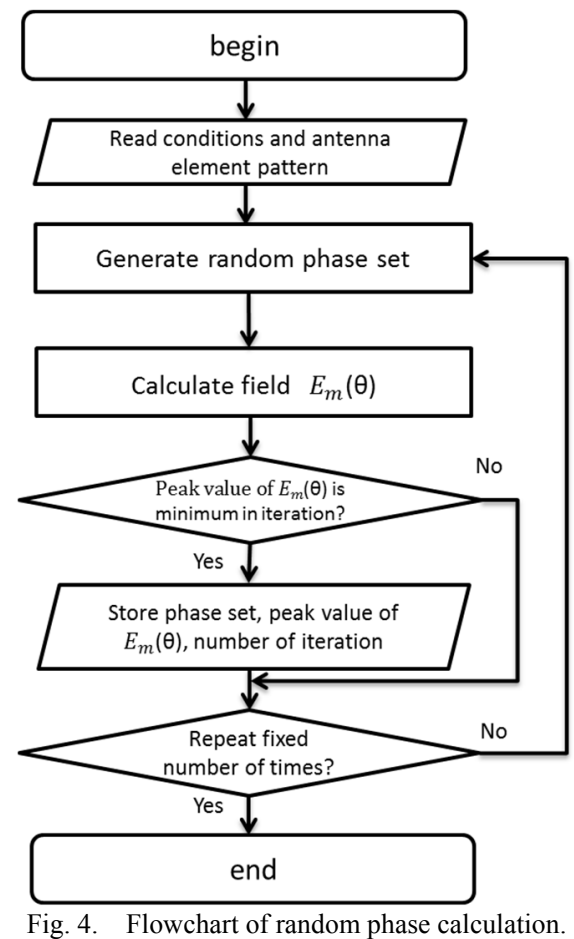

\section{Calculation Method of Random Phase}

Figure 4 shows flowchart of the random phase calculation. In the initial condition, number of elements, number of iteration and antenna element pattern are assumed. In this calculation, number of element is changed from 5 to 100 . If we increase iteration number at large number of element, calculation time increases, so, iteration number is decided experimentally $4^{\text {int } \sqrt{n}}+100$ where $n$ is number of elements.

Next, field $E(\theta)$ is caluculated for all $\theta$, and peak value of $E(\theta)$ is detected. It is compared to the result of previous calculation. If $E(\theta)$ is minimum in all iteration, store the phase set and peak value of $E(\theta)$. This generation of random phase procedure is repeated number of iteration. So, at last, minimum value of $E(\theta)$ is stored. In this paper, we tried to simultaneous degradation of $2 \mathrm{nd}$ and $3 \mathrm{rd}$ harmonics, so, we need to evaluate radiation of 2 nd and 3rd harmonics in Eq. (3)

$$
A=\sqrt{w_{2} \times E_{2}\left(\theta_{2}\right)^{2}+w_{3} \times E_{3}\left(\theta_{3}\right)^{2}}
$$

where $\mathrm{A}$ is a evaluate function.

Then we have to give weighs between 2 nd and 3rd harmonics. This weight shows $w_{2}$ and $w_{3}$ in Eq. (3). Values of $\theta$ is different in 2nd and 3rd harmonics, but simultaneous degradation is available using evaluate function $\mathrm{A}$.

Here, value of $w_{2}$ and $w_{3}$ propotional to harmonics generation power of a rectenna element, we choose $w_{3} / w_{2}=$ 0.67 considering the reference ${ }^{8}$, which describe input power versus harmonics power of each order and several kinds of diodes.

In the former consideration of degradation of only 2nd harmonics ${ }^{5,6)}$, binary phase $[0,90]$ (Phase value is indicated at fundamental frequency, hereafter.) is combined to generate 
random rectenna array because it is reverse phase in 2nd harmonics.

In case of simultaneous degradation of 2 nd and $3 \mathrm{rd}$ harmonics, if we use phase set on binary value $[0,90]$ is not become reverse phase at third harmonics frequency.

So, three-value phase $[0,60,90]$ is considered by adding 60 degree phase element, which becomes reverse phase at $3 \mathrm{rd}$ harmonics. Phase relation of each harmonics is shown in Fig. 5.

Dotted line of Fig. 6 shows harmonics degradation level versus number of element in random rectenna array compared to uniform rectenna array using Eq. (3).

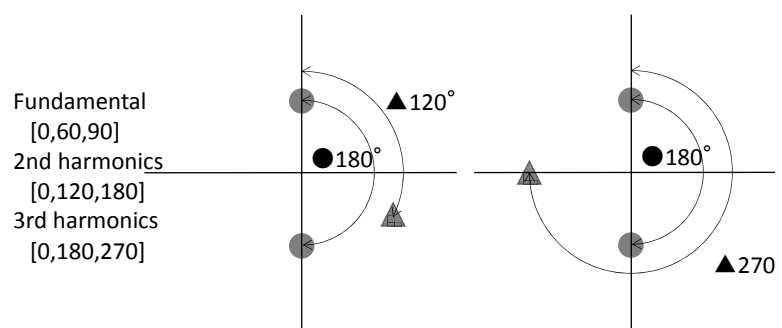

Fig. 5. Three-value phase $[0,60,90]$ in 2 nd and $3 \mathrm{rd}$ harmonics.

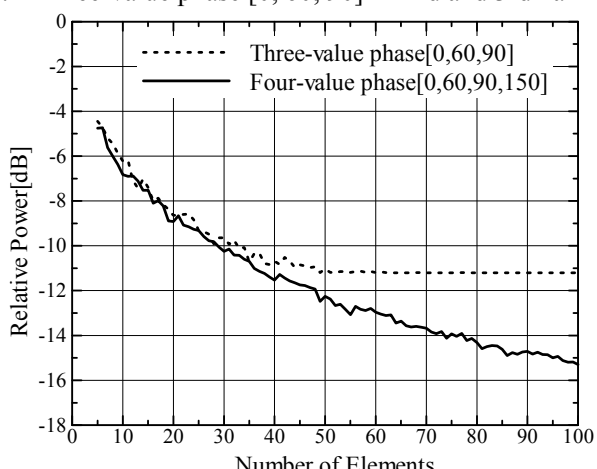

Fig. 6. Harmonics degradation level versus number of element.

Degradation level of three-value phase random rectenna array cannot shows lower than $-11.2 \mathrm{~dB}$ in more than 50 element and its level becomes constant. Because grating lobe of around 0 degree is generated and it cannot remove if we increase the number of element.

So, we increase one more phase of rectenna array, four-value phase $[0,60,90,150]$ is considered for random rectenna array. Figure 7 shows a phase relation of each harmonics.

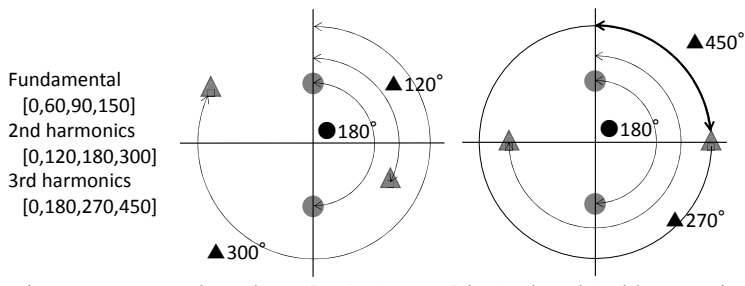

Fig. 7. Four-value phase $[0,60,90,150]$ in 2 nd and 3rd harmonics.

If we use four-value phase, reverse phase combination for all of the phase is generated in 2nd and 3rd harmonics frequencies. Result is shown in solid line of Fig. 6. Degradation level can be improved even in more than 50 elements, and grating lobe of around 0 degree is not observed.

Figure 8 shows angular pattern of 100 element linear array in 2nd harmonics of random rectenna array and conventional in- phase rectenna array. Vertical axes is normalized maximum value of conventional rectenna array.

Random rectenna array has averaged peak in the angular pattern with the maximum $-15.2 \mathrm{~dB}$ at 80 degrees, besides conventional rectenna array has strong peak value around 0 , \pm 45 degrees.

Next, angular pattern of 3rd harmonics of 100 element array is shown in figure 9. Again, even in 3rd harmonics, random rectenna array has averaged peak pattern and its maximum value is $-15.4 \mathrm{~dB}$ at 55 degrees.

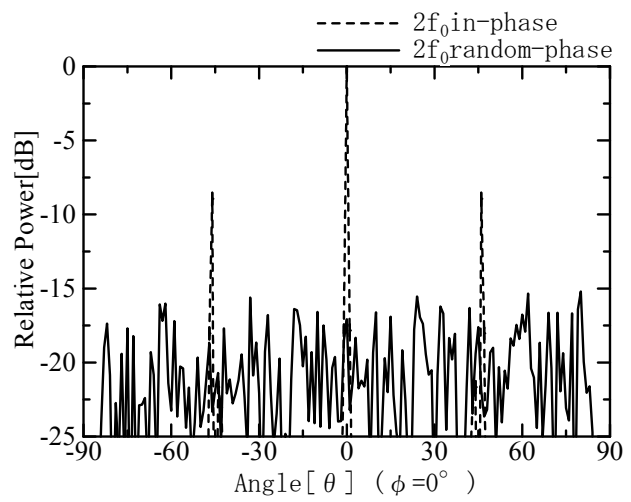

Fig. 8. Pattern of 2 nd harmonics rectenna (100 elements)

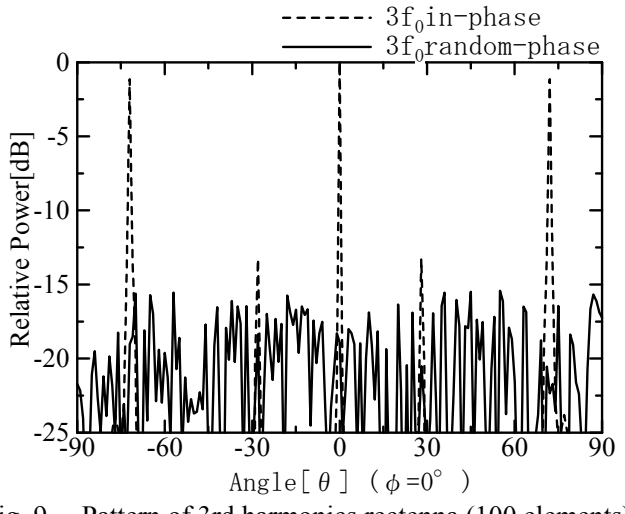

Fig. 9. Pattern of 3 rd harmonics rectenna (100 elements).

\section{Random rectenna array using CMSA}

In this section, we will discuss more realistic situations of random rectenna array, so, CMSA (circular microstrip antenna) is assumed for the rectenna element. It is widely used element and has characteristics of not to resonant harmonics frequencies. So, CMSA is effective for suppression of harmonics reradiation ${ }^{9)}$.

Radiated field of CMSA is shown in next Eq. $(4 \mathrm{~A})(4 \mathrm{~B})^{10)}$.

$$
\begin{aligned}
g_{\theta}(\theta, \phi)= & j^{m^{\prime}} B_{c}\left\{\frac{\exp \left(-j k_{0} R\right)}{R}\right\} \cos \left(m^{\prime} \phi\right) \times \\
& \left\{J_{m^{\prime+1}}\left(k_{0} a \sin \theta\right)+J_{m^{\prime-1}}\left(k_{0} a \sin \theta\right)\right\} \\
g_{\varphi}(\theta, \phi)= & j^{m^{\prime}} B_{c}\left\{\frac{\exp \left(-j k_{0} R\right)}{R}\right\} \cos \theta \sin \left(m^{\prime} \phi\right) \times \\
& \left\{J_{m^{\prime+1}}\left(k_{0} a \sin \theta\right)+J_{m^{\prime}-1}\left(k_{0} a \sin \theta\right)\right\}
\end{aligned}
$$

where $B_{c}=\frac{V_{0} a k_{0}}{2}, m$ ' and $n$ ' shows mode number of CMSA $\mathrm{TM}_{m \prime n^{\prime}} . V_{0}$ is maximum voltage at open boundary in CMSA and express $V_{0}=t E_{0} J_{m^{\prime}}\left(X_{m^{\prime} n^{\prime}}\right) . X_{m^{\prime} n^{\prime}}$ is n'th root of 
$J_{m^{\prime}}^{\prime}(k a), a$ and $t$ shows patch radius and thickness, respectively.

Radiation pattern of 2 nd harmonics is calculated using $T M_{010}$ mode, because it is nearest mode in frequency. Also, radiation pattern of 3 rd harmonics is calculated using $T M_{120}$ mode, in the same reason.

In this calculation, fundamental frequency is assumed 5.85 $\mathrm{GHz}$, maximum number of element is 100 , and patch radius is $\mathrm{a}=9.96[\mathrm{~mm}]$, substrate thickness and permittivity is $\mathrm{t}=0.762[\mathrm{~mm}]$ and $\varepsilon_{r}=2.16$, respectively. Also, iteration set to $1,000,000$ times for each antenna element. A reflection loss of CMSA is compensated using 9).

Calculation algorithm shown in Fig. 4 and four-value phase $[0,60,90,150]$ is used. Figure 10 illustrates calculation

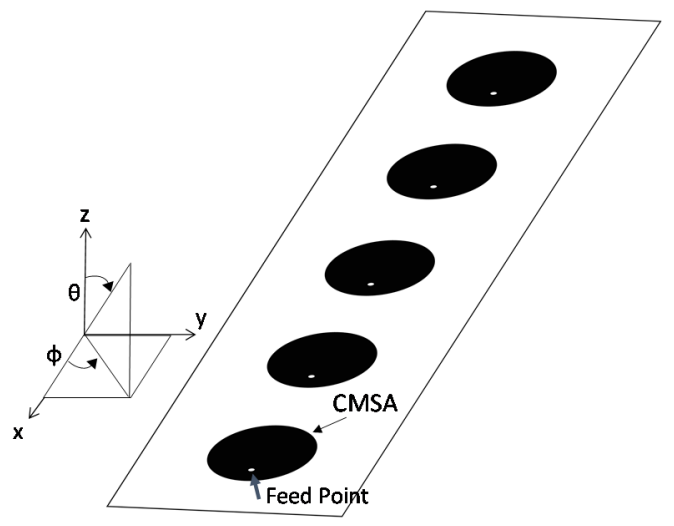

Fig. 10. CMSA rectenna array model.

model of CMSA array rectenna, which is 5 elements linear array for example. CMSA arranged in E-plane, so, xz-plane pattern is calculated.

Figure 11 shows the harmonics degradation level versus number of element in CMSA using Eq. (3).

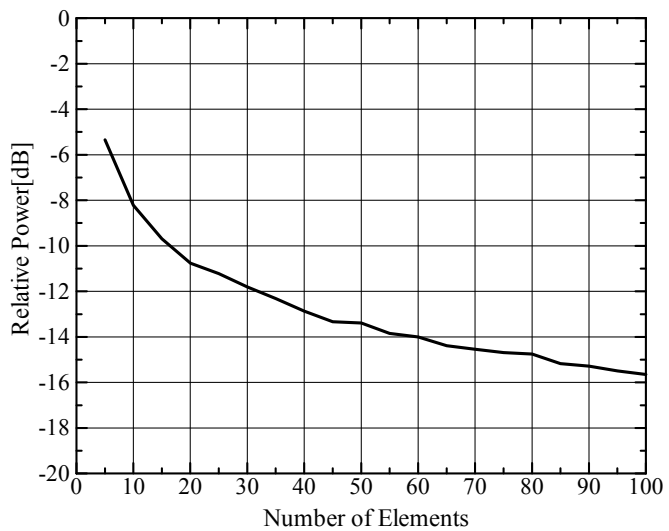

Fig. 11. Harmonics degradation level versus number of element in CMSA (linear array).

2nd harmonics pattern of CMSA random rectenna arrray of 100 elements are shown in Fig. 12 compared with conventional array. In this pattern, conventional rectenna array shows the strong peak around 45 degree, but random rectenna array does not shows peak value in all angle. Moreover, null is observed around 0 degree. In this angle element pattern of 2 nd harmonics of CMSA becomes to null, so, field pattern also shows centernull. From this pattern, $-15.8 \mathrm{~dB}$ degradation of harmonics is observed. 3rd harmonics pattern of CMSA random rectenna array of 100 elements are shown in Fig. 13. Conventional rectenna array also shows strong peak, and random rectenna array observed null at 30 degree. It is null point of CMSA element pattern of $3 \mathrm{rd}$ harmonics. And $-15.0 \mathrm{~dB}$ degradation is observed at third harmonics.

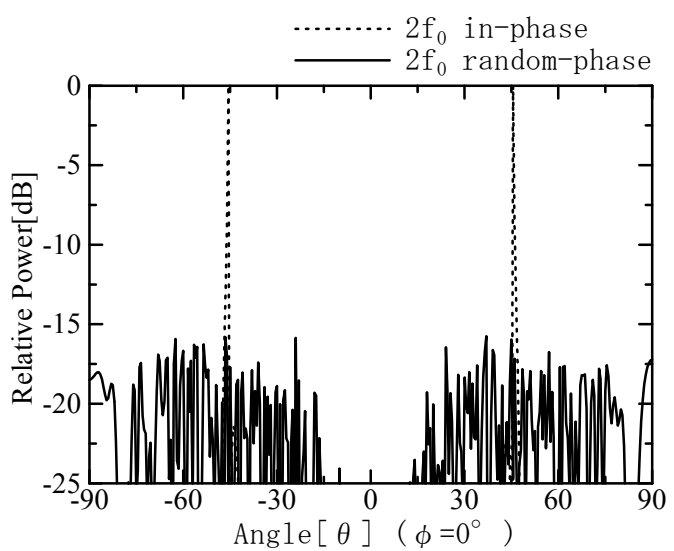

Fig. 12. CMSA rectenna array pattern of 2 nd harmonics.

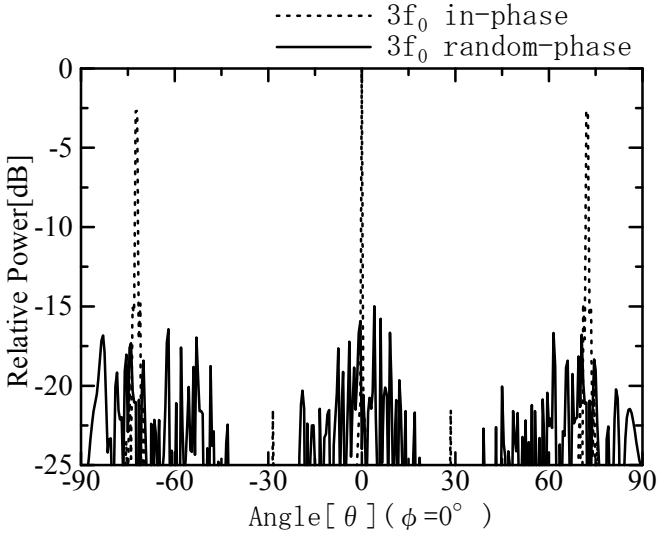

Fig. 13. CMSA rectenna array pattern of $3 \mathrm{rd}$ harmonics.

Next, we calculated in the case of 2-dimensinal array rectenna that assumed 25 elements CMSA and 5x5 rectangular arrangement. Inter-element distance is $0.7 \lambda$, weight ratio of $w_{3} / w_{2}=0.67$ is also assumed. The calculation in the two dimensional takes time very much compared to one dimensional case. So, iteration number is fixed to 100,000 .

Re-radiation pattern of 2nd and 3rd harmonics are shown in Fig. 14 and Fig. 15, respectively. Degradation level of 25 element 2D rectenna array evaluated using Eq. (3) becomes $-7 \mathrm{~dB}$, which can assume more degradation level compared to one- dimensional 5 element case.

\section{Conclusion}

In order to suppress the harmonics reradaiation from rectenna array, simultaneous degradation of 2 nd and $3 \mathrm{rd}$ harmonics is tried using random rectenna array.

Usage of four-value phase in the array element phase is proposed and its validity is confirmed by calculation for the simultaneous degradation of 2 nd and 3rd harmonics.

Three numerical examples are calculated. In 100 elements 


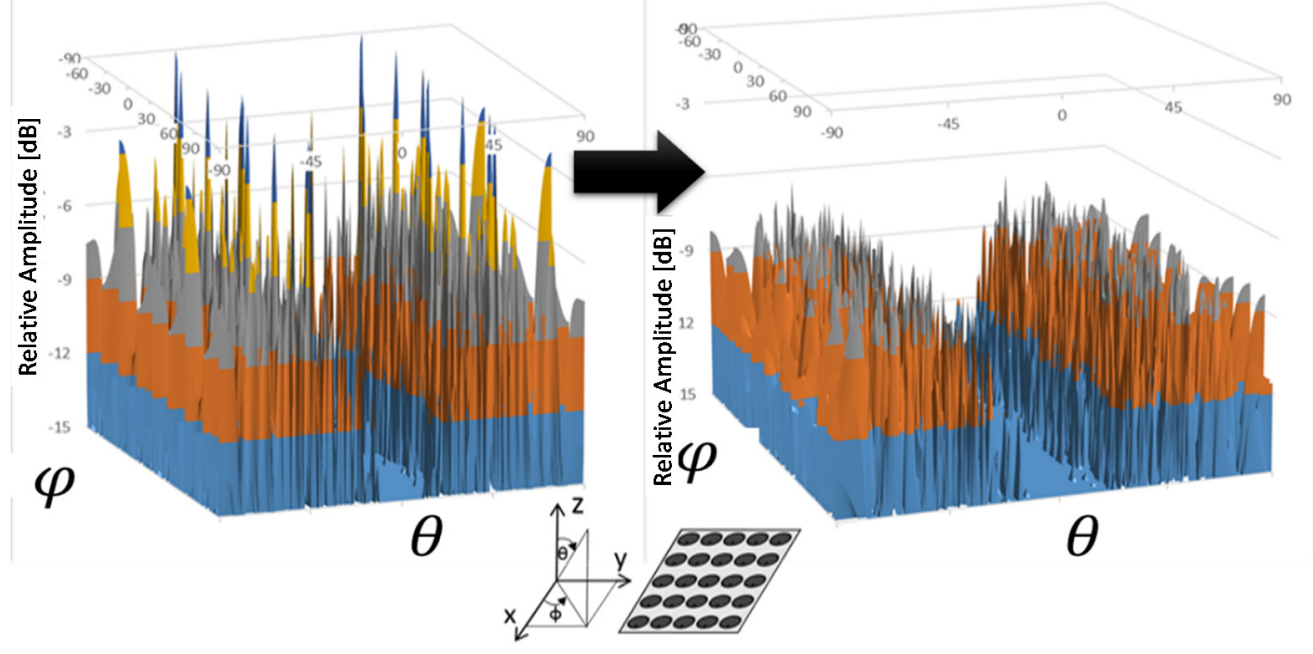

(a) in-phase

(b) random phase

Fig. 14. 2nd harmonics pattern of 2D-CMSA rectenna (25 elements).

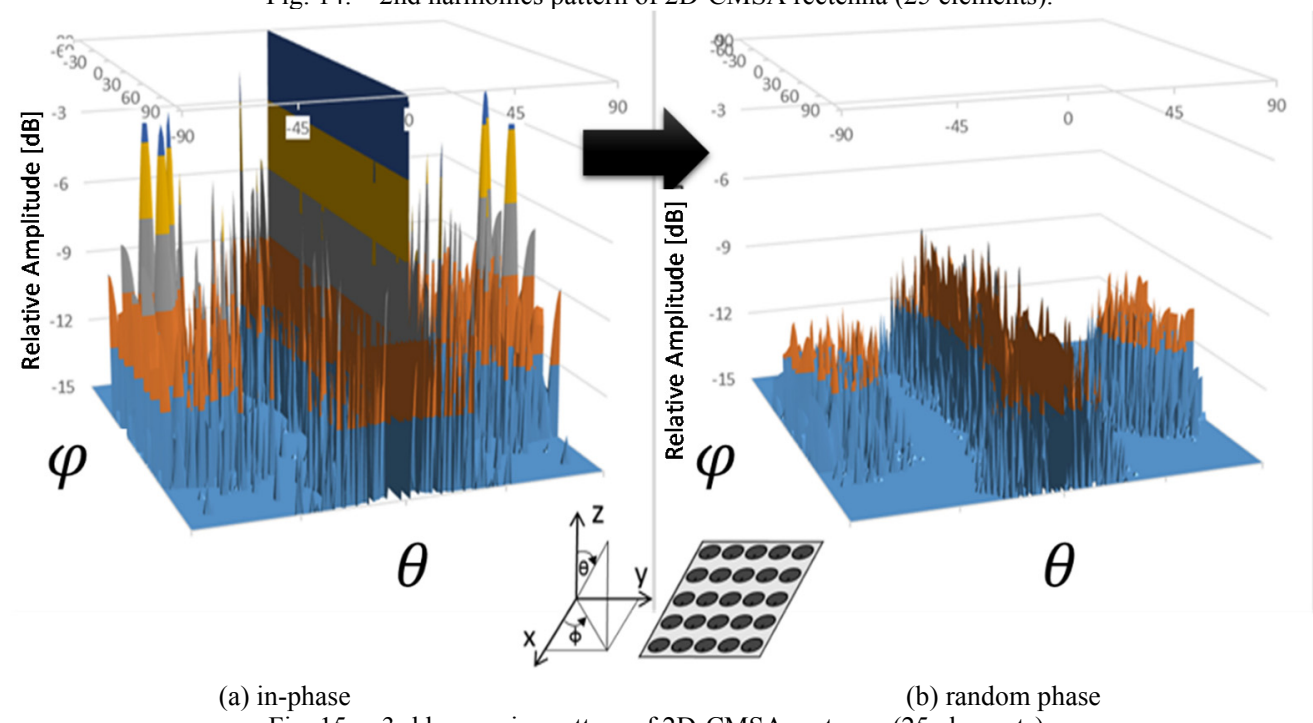

Fig. 15. 3rd harmonics pattern of 2D-CMSA rectenna ( 25 elements).

linear array both omni-directional and CMSA array shows $-15 \mathrm{~dB}$ degradation and 25 elements 2D CMSA array shows $-7 \mathrm{~dB}$ degradation level.

The proposed method will be one candidate to solve the EMC problem with the realization of microwave SSPS.

\section{References}

1) Sugiura, A.: Environmental problem on SPS, 1. On ground EMC problem from SPS, Review of Radio Research Lab., 28 (1982), pp.669-687 (In Japanese).

2) Fujino, Y. and Fujita, M.: A measurement method and its evaluation of re-radiation of harmonic waves from a planar rectenna, IEICE Trans on Commun., J81-B-II (1998), pp.112-118 (In Japanese).

3) Fujino, Y. and Fujita, M.: An experimental study for evaluating reradiation of higher harmonic waves from a rectenna for microwave power reception, Trans., IEE of Japan, 117-A (1997), pp.490-495 (In Japanese)

4) Radchenko, V. and Fujita, M.: Rectenna array synthesis for electromagnetic compatibility improvement, Space Energy and
Transportation, 1 (1996), pp.443-457.

5) Fujino, Y. and Suzuki, K.: A study of harmonics re-radiation from a rectenna -Fundamental consideration toward a rectenna array-, Technical report of IEICE, SPS2005-04 (2005), pp.19-22, (In Japanese).

6) Suzuki, K., Fujino, Y., Furukawa, M. and Fujiwara, T.: Fundamental study of a rectenna array with harmonics suppressing function for Solar Power Satellite, Technical report of IEICE, SPS2005-04 (2006), pp.21-25 (In Japanese).

7) Otsuka, M., Omuro, N., Kakizaki, K., Saitoh, S., Kuroda, M., Horiuchi, K and Soejima, T.: Relation between spacing and receivimg efficiency of finite rectenna array, IEICE Trans. Commun., J73-B-II (1990), pp.133-139 (In Japanese).

8) Fujino., Y. and Fujita, M.: Measurement of reradiation of micowaves from a rectenna, Review of Communications Radio Research Lab., 44 (1998), pp.161-174 (In Japanese).

9) Syahrial, Tamura, N., Yamada, Y., Omiya, M. and Itoh, K.: Considerations on a circular microstrip patch antenna with slits for rectenna elements, IEICE Trans. on. Comm., J84-B (2001), pp.244253 (In Japanese).

10) Haneishi, M., Hirasawa, K. and Suzuki, Y., Small Planar Antenna, IEICE, Tokyo, 1996, pp.112-116 (In Japanese). 